\title{
Soil Image Segmentation and Texture Analysis: A Computer Vision Approach
}

\author{
Anastasia Sofou, Student Member, IEEE, Georgios Evangelopoulos, Student Member, IEEE, and \\ Petros Maragos, Fellow, IEEE
}

\begin{abstract}
Automated processing of digitized soilsection images reveals elements of soil structure and draws primary estimates of bioecological importance, like ground fertility and changes in terrestrial ecosystems. We examine a sophisticated integration of some modern methods from computer vision for image feature extraction, texture analysis, and segmentation into homogeneous regions, relevant to soil micromorphology. First, we propose the use of a morphological partial differential equation-based segmentation scheme based on seeded region-growing and level curve evolution with speed depending on image contrast. Second, we analyze surface texture information by modeling image variations as local modulation components and using multifrequency filtering and instantaneous nonlinear energy-tracking operators to estimate spatial modulation energy. By separately exploiting contrast and texture information, through multiscale image smoothing, we propose a joint image segmentation method for further interpretation of soil images and feature measurements. Our experimental results in images digitized under different specifications and scales demonstrate the efficacy of our proposed computational methods for soil structure analysis. We also briefly demonstrate their applicability to remote sensing images.
\end{abstract}

Index Terms-Computer vision, image segmentation, remote sensing, soil analysis, texture analysis.

\section{INTRODUCTION}

$\mathbf{I}$ MAGE data in geosciences are common and require processing and measurement schemes that range from small microscopic scales to large remote sensing scales. In this work, we focus mainly to the first category and specifically in images of thin soilsections. The goal of soil micromorphology, as a branch of soil science, is the description, interpretation, and measurement of components, features, and fabrics in soils at a microscopic level. Basic soil components are the individual particles (e.g., quartz grains, clay minerals, plant fragments) that can be resolved with the optical microscope together with the fine material that is unresolved into discrete individuals. Soil structure is concerned with the size, shape, sharpness, contrast, frequency, and spatial arrangement of primary particles and voids. Many of these characteristics are a function of the orientation of components and the direction in which they are cut as well as of the magnification used. Soilsection images produced via a digitizing procedure, using conventional scanners,

Manuscript received November 15, 2004; revised March 22, 2005. This work was supported in part by the Greek GSRT Research program PENED-2001 and the European NoE MUSCLE and in part by the Greek research program "Pythagoras" and the National Technical University of Athens research program "Protagoras."

The authors are with the School of Electrical and Computer Engineering, National Technical University of Athens, 15773 Athens, Greece (e-mail: sofou@cs.ntua.gr; gevag@cs.ntua.gr; maragos@cs.ntua.gr).

Digital Object Identifier 10.1109/LGRS.2005.851752 cameras, or microscopes under polarized light, exhibit a great variety of geometric features. Important image features that provide useful information for soil structure quality evaluation include cluster/particle shape, either one-dimensional (1-D), such as edges or curves, or two-dimensional (2-D), such as light or dark blobs (small homogeneous regions of random shape), spatial arrangement of soil components, and their texture.

The current analysis used in soilsection images is basically confined to thresholding, histogram measurements, simple digital signal processing techniques and fractal dimension estimation [1], [2]. Our general long-term research goal is to surpass the aforementioned techniques and integrate modern computer vision methodologies such as image segmentation and texture analysis toward a high-level system capable of analyzing and evaluating soilsection fertility and bioecological quality [3]. In effect, the expensive and time-consuming process of traditional biochemical analysis will be automated, enhanced by, and possibly coupled with computer vision techniques.

The research work presented here aims at coupling image segmentation and texture analysis in the soilsection image framework. Specifically, in the next sections we deal with the segmentation of soil images into distinctive regions (crystals, opaque material, organic matter, void spaces) employing nonlinear morphological tools, curve evolution, and partial differential equations (PDEs) methods. Additionally, by using appropriate texture modeling, we propose efficient and well-motivated extraction of features capable of quantifying texture characteristics like roughness, geometrical complexity, rate of change in local contrast variations, and orientation. Finally, we couple the most prominent features of soilsection images, color/intensity, geometry, and texture, and utilize them so as to correctly lead the final segmentation process. The presented methods can be extended to broader classes of geoscience data and applied in general remote sensing surfaces.

\section{IMAGE SEgMENTATION}

Image segmentation is an important yet difficult computer vision task, as it requires to some extent a semantic understanding of the image. Generally speaking, it is the process of partitioning the image into disjoint regions, each one being homogeneous and connected with respect to some property, such as gray-value, color, texture, motion. It can be divided into three different but complementary stages, i.e., 1) preprocessing; 2) region/feature extraction; and 3) segmentation algorithm application, each one being significant for the final segmentation result.

Amongst the variety of segmentation methods, the morphological watershed transform has proved to be very powerful and effective, especially when coupled with nonlinear multiscale morphological operators [4], [5]. The underlying idea is the following: 1) a gradient image of the scene is constructed; 
2) for each object of interest or homogeneous region a marker (set of feature points inside desired region) is detected; and 3 ) the watershed lines associated to the markers are constructed. Watershed transform can be topographically described as a flooding process, where the image function is considered as a topographic surface immersed in water. The markers serve as flooding sources, from where waves start emanating forming various lakes. At points where different waves meet, a dam is erected to avoid lake merging, which is in fact the watershed line that separates the image into different regions. In mathematical morphology, flooding has been implemented via immersion simulations [5] and hierarchical queues [4].

Apart from the morphological flooding approach, watershed has also been modeled in a continuous way via the eikonal (PDE) [6], [7], usually employing ideas from the field of curve evolution [8]. Motivations for using PDEs include better and more intuitive mathematical modeling, connections with physics, and better approximation to the continuous geometry of the problem. Using PDE modeling in the flooding process of watershed transform, each emanating wave's boundary is viewed as a curve, which evolves with predefined speed. Specifically, the boundary of the marker is considered as a moving smooth closed curve $\vec{C}(p, t)$ where $p \in[0,1]$ parameterizes the curve and $t$ is an artificial marching parameter. The PDE that implements the watershed flooding, as well as its level set approach [9] where the evolving curve is embedded as the zero level set $\Gamma(t)=\{(x, y): \Phi(x, y, t)=0\}$ of a higher dimension space-time function $\Phi(x, y, t)$, are given by

$$
\begin{aligned}
\frac{\partial \vec{C}}{\partial t} & =\frac{c}{A(t)\|\nabla I\|} \cdot \vec{N} \\
\frac{\partial \Phi}{\partial t} & =\frac{c}{A(t)\|\nabla I(x, y)\|}\|\nabla \Phi\| .
\end{aligned}
$$

$A(t)$ is either 1 if we perform only contrast-based segmentation (height flooding) or $A(t)=\operatorname{Area}(\vec{C})$ in case of contrast and size segmentation (volume flooding) [10].

Efficient algorithms [11] to solve time-dependent eikonal PDEs are the narrow-band level sets and the fast marching method (for stationary formulations of eikonal PDEs).

\section{TEXTURE ANALYSIS}

Image texture refers to patterns caused by contrast variations and an inherent inhomogeneity in natural surfaces, resulting from properties such as roughness, depth, illumination, color, etc. Texture analysis in computer vision aims at the problems of feature extraction, segmentation and classification, synthesis, and inferring shape from texture.

Elementary natural texture components can be interpreted as locally smooth modulations and hence assumed nonstationary signals, well localized within a narrow band in the spatial frequency plane. Textured surfaces can be modeled by a sum of 2-D spatial amplitude and frequency modulation (AM-FM) signals

$I(x, y)=\sum_{k=1}^{K} a_{k}(x, y) \cos \left[\phi_{k}(x, y)\right], \quad \vec{\omega}_{k}(x, y)=\nabla \phi_{k}(x, y)$

where each of the $K$ components is a 2-D nonstationary sine with a spatially varying amplitude $a_{k}(x, y)$ and a spa- tially varying instantaneous frequency vector $\vec{\omega}_{k}(x, y)=$ $\left(\omega_{1}(x, y), \omega_{2}(x, y)\right)$. The amplitude models local contrast variations, and the frequency vector contains rich information about the locally emergent spatial frequencies [12], [13].

For the estimation of the 2-D modulation signals, an approach of low complexity and small estimation error was developed in [14] based on an energy operator $\Psi(f) \triangleq\|\nabla f\|^{2}-f \nabla^{2} f$, which is a multidimensional extension of the 1-D Teager energy operator. Applying $\Psi$ to a 2-D AM-FM signal $f(x, y)=$ $a_{k}(x, y) \cos \left[\phi_{k}(x, y)\right]$, modeling a texture component, yields

$$
\Psi\left[a_{k} \cos \left(\phi_{k}\right)\right] \approx a_{k}^{2}\left\|\vec{\omega}_{k}\right\|^{2} .
$$

The product in (4), which couples the squares of the instantaneous amplitude and frequency magnitude, may be called the texture modulation energy. By the assumption that the instantaneous amplitude and frequency do not vary rapidly in space or too greatly in value compared with the carriers, the above approximation error becomes negligible. A decoupling of this energy, in the two modulation signals, is possible by also applying the energy operator on the image component derivatives $\partial f / \partial x$ and $\partial f / \partial y$, via a nonlinear algorithm called energy separation algorithm (ESA) [14]

$$
\begin{aligned}
& \sqrt{\frac{\Psi\left(\frac{\partial f}{\partial x}\right)}{\Psi(f)}} \approx\left|\omega_{k 1}(x, y)\right| \sqrt{\frac{\Psi\left(\frac{\partial f}{\partial y}\right)}{\Psi(f)}} \approx\left|\omega_{k 2}(x, y)\right| \\
& \frac{\Psi(f)}{\sqrt{\Psi\left(\frac{\partial f}{\partial x}\right)+\Psi\left(\frac{\partial f}{\partial y}\right)}} \approx\left|a_{k}(x, y)\right| \text {. }
\end{aligned}
$$

A unique, spatially varying AM-FM texture component is thus characterized at each location by the estimated amplitude envelope and magnitude of instantaneous frequencies.

The AM-FM models are not applied directly to an image, but instead they are used on its bandpass filtered versions [12]. Isolation of modulation components requires filtering mechanisms with sufficient spatial and spectral localization, usually done by filterbanks spanning various radial frequencies and orientations. For the generating kernel, Gabor filters are an optimal choice, following biological mechanisms, being compact and smooth and attaining the lower limit of joint space-frequency resolution uncertainty. Two-dimensional Gabor filters are characterized by impulse responses of the form $h_{k}(x, y)=\exp \left[-(x / \alpha)^{2}-\right.$ $\left.(y / \beta)^{2}\right] \cos \left(u_{k} x+v_{k} y\right)$, where $(2 \pi \alpha)^{-1},(2 \pi \beta)^{-1}$ are the rms bandwidths in each dimension, and $\left(u_{k}, v_{k}\right)$ are the $k$ th filter's central frequency coordinates.

Representations indicative of the dominant texture components are obtained by an energy-tracking mechanism in the multidimensional feature space consisting of the filter responses. The filtered texture components are subjected to energy measurements via the 2-D energy operator $\Psi$ and demodulation through the ESA algorithm ( 5). The energies are then averaged by a local averaging filter $h_{a}$ and are subjected to pixelwise comparisons. The filter with the maximum average Teager energy, given by

$$
\Psi_{\text {mat }}(I(x, y))=\max _{k} \Psi\left[\left(\left(I * h_{k}\right) * h_{a}\right)(x, y)\right]
$$

where $*$ denotes 2-D signal convolution, indicates the most prominent texture component. The derived $\Psi_{\text {mat }}$ is a slowly varying descriptor of texture modulation energy, which can 
indicate various energy levels and thus different textures with respect to their Teager energy signatures. In parallel, a low-dimensional feature set is obtained by terming the dominant modulation amplitude $\alpha_{D}(x, y)$ and frequency vector $\vec{\omega}_{D}(x, y)$ as the set of demodulated values corresponding in each image location to the most active, in the above energy sense, filter output. The modulation features obtained by tracking the dominant texture components along multiple bands provide both local and global texture information and serve as rich descriptors of a wide variety of textures. They have been recently used with success for texture segmentation in [15].

\section{Coupled Segmentation Scheme}

A recently proposed method for image decomposition is the $I=U+V$ model [16], where the $U$ part is called the "cartoon component" and consists of relatively flat plateaus for the object regions surrounded by abrupt edges, whereas the $V$ part is called "texture oscillation" and contains texture plus noise information. By treating and processing the two components separately, a powerful joint segmentation scheme is proposed. Contrast variations are taken into account from the $U$ part, and texture oscillations are approached through modulation analysis on the $V$ component.

Several nonlinear, edge-preserving, image smoothing schemes can create cartoon approximations of an image such as the anisotropic diffusion or total variation scheme [16]. We use levelings [17], which are nonlinear object-oriented and contour-preserving filters, to simplify an image $I$ by locally expanding/shrinking an initial seed image, called the marker $M$, and globally constraining the marker evolution by the reference image. Specifically, iterations of the image operator $\lambda(F \mid I)=(\delta(F) \wedge I) \vee \varepsilon(F)$, where $\delta(F)$ and $\varepsilon(F)$ are dilation and erosion, respectively of $F$ by a small disk, yield in the limit the leveling of $I$ w.r.t. $M$, denoted as $\Lambda(M \mid I)=\lim _{k \rightarrow \infty} F_{k}$, $F_{k}=\lambda\left(F_{k-1} \mid I\right), F_{0}=M$.

We set as cartoon component the leveling $U=\Lambda(M \mid I)$ and as texture component the residual $V=I-U$. We construct multiscale leveling cartoons $U_{i}$ from a sequence of multiscale markers $M_{i}$, obtained from sampling a Gaussian scalespace. The corresponding residuals $V_{i}=I-U_{i}$ constitute a hierarchy of multiscale texture components. As an alternative marker, we consider the use of anisotropic diffusion, where at each sequence step the leveling marker is obtained by a version of the image with blurred regions but adequately preserved boundaries, caused by the constrained diffusion process.

The proposed new segmentation scheme is based on the following curve evolution PDE:

$$
\frac{\partial \vec{C}}{\partial t}=\left(\frac{\lambda_{1}}{A(t)\left\|\nabla f_{1}\right\|}+\lambda_{2} \Psi_{\text {mat }}\left(f_{2}\right)-\mu \kappa\right) \vec{N}
$$

where $f_{1}$ and $f_{2}$ are image transformations related to the original $I$, but not necessarily the same. Thus, the curve's speed depends on three terms: the first two are eikonal, whereas the third (curvature motion) is diffusive. All terms are linked with some optimality criterion. The first term drives the curve with speed that maximizes the flooding of the $f_{1}$ image toward its watershed. The second term can be shown to correspond to a flow that maximizes the average texture energy: $\max \iint_{R(C)} \Psi(f) \Longrightarrow$ $\partial \vec{C} / \partial t=\Psi(f) \vec{N}$. This term pushes the curve toward regions with large texture energy.
Following the level set formulation in [9], we embed this evolving planar curve as the zero-level curve of an evolving space-time function $\Phi(x, y, t)$

$$
\frac{\partial \Phi}{\partial t}=\left(\frac{\lambda_{1}}{A(t)\left\|\nabla f_{1}\right\|}+\lambda_{2} \Psi_{\text {mat }}\left(f_{2}\right)-\mu \operatorname{curv}(\Phi)\right)\|\nabla \Phi\|
$$

where $\operatorname{curv}(\Phi)$ is the curvature of the level sets of $\Phi$.

Based on the PDE (7), different scenarios can be obtained by varying the signals $f_{1}$ and $f_{2}$. The most obvious choice is $f_{1}=$ $I, f_{2}=I$, but we also propose another novel and promising scenario, which is $f_{1}=U, f_{2}=V$. The former is a curve evolution with velocity inversely proportional to the intensity contrast (or volume) of the input image and proportional to the $\Psi_{\text {mat }}$ energy of the image. The latter is a curve evolution with speed inversely proportional to the intensity contrast (or volume) of the cartoon component and proportional to the $\Psi_{\text {mat }}$ energy of the textured component, and is favored and further investigated since it integrates edge and texture information by combining the different signals produced by the $U+V$ decomposition of the image.

In the proposed scheme, there are multiple curves to be propagated, initialized as the contours of a marker set, indicative of significant/homogeneous image regions. In general, marker extraction is application dependent and there is a great variety of methodologies dealing with this problem. Thus, markers can be: 1) contrast-oriented corresponding to peaks or valleys of certain depth (obtained via reconstruction filters); 2) peaks of $\Psi_{\text {mat }}(I)$, indicating areas with rich texture; 3 ) combination of contrast as well as texture criteria; and 4) manually placed at areas of interest. The implementation of marker contour propagation (8) has been done with established techniques from level sets methods. If $\mu=0$ the PDE is of pure eikonal-type, and its implementation is based on the fast marching methodology (FMM) [11], which ensures computational speed. If $\mu \neq 0$ the PDE is implemented using the narrow-band method [11], and the segmentation boundaries are smoothed. The use of the $\kappa$-term is optional.

\section{EXPERIMENTS AND DISCUSSION}

The proposed methods for texture analysis, decomposition, and joint segmentation have been applied to large-scale soilsections scanned at $47 \mathrm{pixel} / \mathrm{micron}$ and small-scale ones from microscopes with polarized light at 1 pixel/micron. In Fig. 1, we present an example of the second category, where multiband texture modulation energy tracking is applied and the set of dominant features are extracted. In Fig. 1(a), we can clearly discriminate organic and nonorganic matter (large gray and white objects), voids (black areas), and fine structure clusters. The well-localized texture energy measurement in Fig. 1(c) indicates strong texture variations. This type of dual, amplitude and frequency, information jointly captured by the $\Psi_{\text {mat }}(I)$ energy supports the use of modulation energy for texture analysis. Spatial resolution is almost excellent with operations on $3 \times 3$ local window, and spectral depending on the filters' bandwidth.

To evaluate texture analysis effectiveness in terms of classification, independently of the classifier choice, we consider the separability of classes in a set of typical soil textures. The Fisher criterion [18] is a measure of intercluster distance and compactness. According to the Fisher linear discriminant, feature vectors corresponding to two different classes are linearly projected to the 1-D space to achieve maximum separability, given by the 

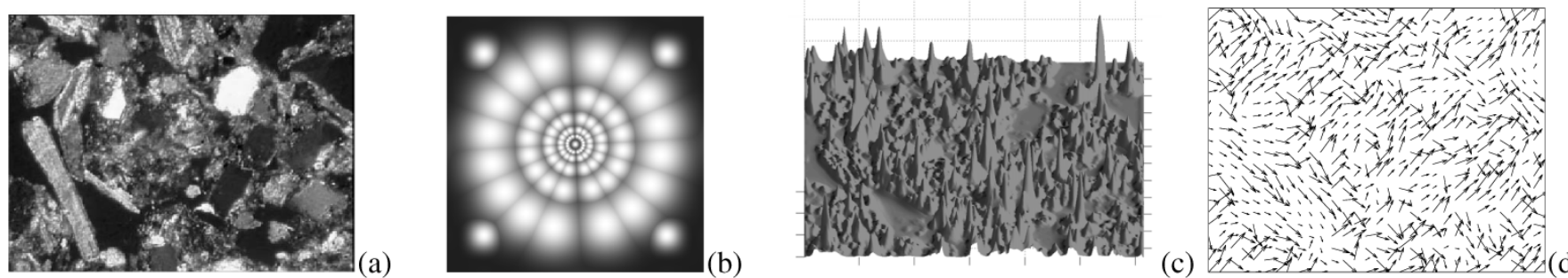

Fig. 1. Texture analysis. (a) Soilsection image $(768 \times 567$ pixels $)$ digitized at 1 pixel $/$ micron. (b) Gabor filterbank frequency responses. (c) Perspective view of maximum average Teager energy. (d) Dominant frequency orientation vectors.
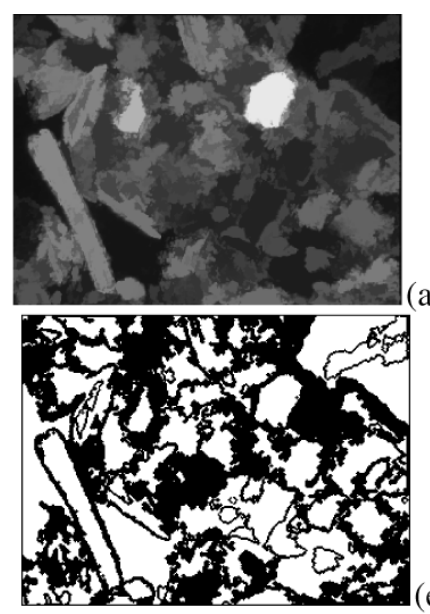
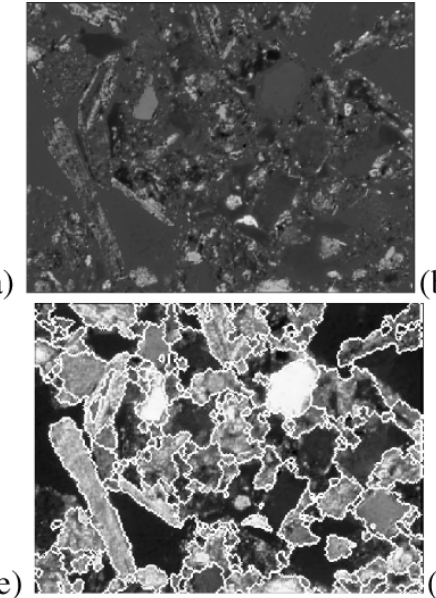
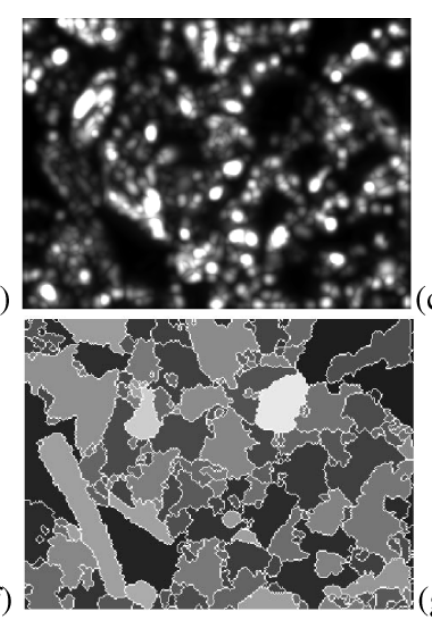
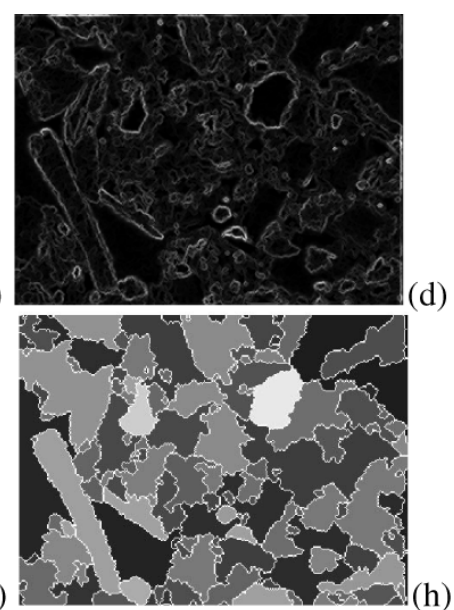

Fig. 2. Segmentation results. (a) "Cartoon component" $U$. (b) "Texture component" $V$. (c) Texture energy $\Psi_{\text {mat }}(V)$ ). (d) Gradient magnitude. (e) Markers. (f) Coupled segmentation scheme result $(A(t)=1)$. (g) Segmentation regions. (h) Refined segmentation regions.
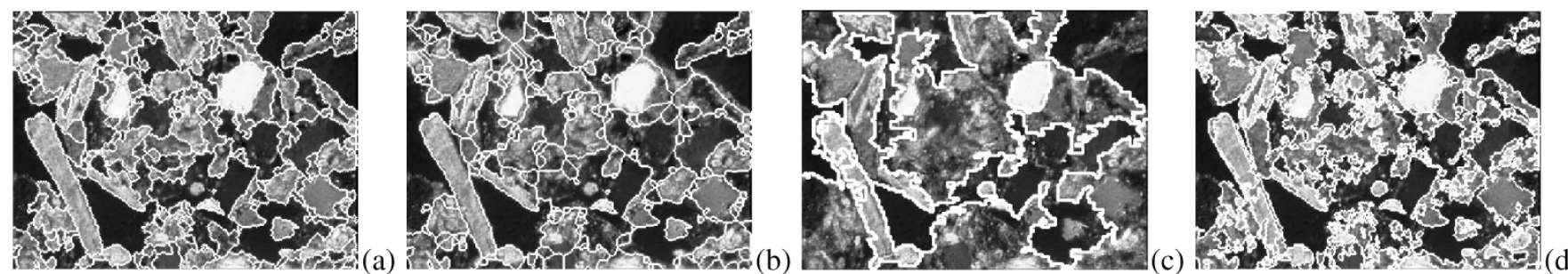

Fig. 3. Different segmentation methods results and the their corresponding goodness measures LYGC and MSF. (a) Coupled segmentation scheme results $(A(t) \neq$ 1 ), $\mathrm{LYGC}=2.24, \mathrm{MSF}=1.45$. (b) Watershed segmentation, $\mathrm{LYGC}=4.04, \mathrm{MSF}=1.47$. (c) Split and merge segmentation based on RSST, LYGC $=35.5$, $\mathrm{MSF}=22.3$. (d) K-means clustering segmentation, $\mathrm{LYGC}=4.49, \mathrm{MSF}=2.10$. For the result of Fig. $2(\mathrm{f}) \mathrm{LYGC}=2.51, \mathrm{MSF}=1.46$.

criterion $f=\left|\mu_{1}-\mu_{2}\right| / \sqrt{\sigma_{1}^{2}+\sigma_{2}^{2}}$, depending on the distance of the means relative to the sum of variances of the projected distributions.

We consider three feature vectors to compare a wellestablished multiband method with the proposed: 1) Gabor energy features [18], [19], the quadrature filter magnitude of every band; 2) Teager energy of the Gabor responses; and 3) modulation energy $\Psi_{\text {mat }}$, dominant frequency orientation $\angle \vec{\omega}_{D}$, and image intensity $I$. While the two vectors are of the same dimension per pixel as the number of filters, the dominant and intensity features highly decrease dimensionality. For all pairs of the four typical texture classes in Fig. 4, the Fisher distances attained by the three vectors $f_{g}, f_{t}$, and $f_{d}$, respectively, were computed. The max and min distances with the corresponding texture pairs per method are $f_{g}=$ [1.41 (Q-O1), $1.03(\mathrm{Q}-\mathrm{F})], f_{t}=[1.56(\mathrm{Q}-\mathrm{O} 1), 1.10(\mathrm{Q}-\mathrm{F})]$, $f_{d}=[1.65(\mathrm{~F}-\mathrm{O} 1), 1.01$ (F-O2)]. Gabor Teager energy features attain higher separability for any pair, with a mean
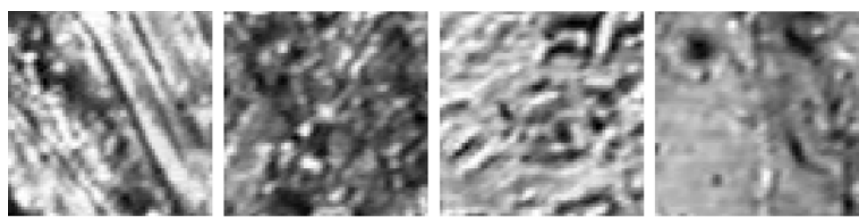

Fig. 4. Typical soil texture details. (Left to right) Quartz grains-crystals (Q), fine matter $(\mathrm{F})$, and organic matter $(\mathrm{O} 1, \mathrm{O} 2)$.

distance of 1.33 , compared to typical energy, of mean 1.23 . The low-dimensionality dominant vector attains the maximum distance and an average 1.27 over all pairs.

Texture modulation energy balances the tradeoff between dimensionality and effectiveness, and the energy operator outperforms the conventional energy measurements. The emerged dominant modulation features can be used for further analysis of segmented soil images, texture classification, or distribution estimation regarding region type, texture, or geometry. 


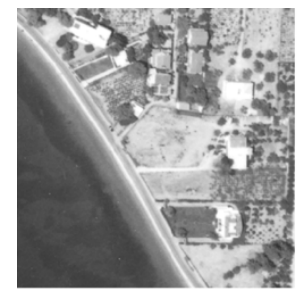

(a)

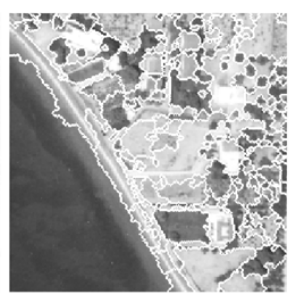

(b)

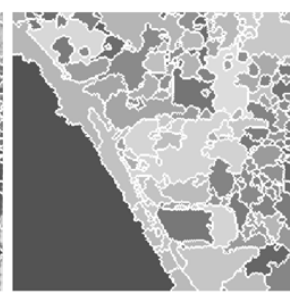

(c)
Fig. 5. Large-scale segmentation results in aerial image detail.

Following the monochromatic $U+V$ decomposition using the leveling approach with anisotropic or Gaussian markers and texture feature extraction [Fig. 2(a)-(c)], an appropriate set of automatically obtained segmentation markers [Fig. 2(e)] is evolved according to the PDE scenario of (8) where $f_{1}=U$ and $f_{2}=V$. The initial segmentation results [Fig. 2(f)-(g)], due to the multistructured nature of these images, are refined by a region postmerging procedure [Fig. 2(h)] based on a similarity measure and a Fisher distance variance constraint.

The proposed scheme was tested against other simpler but established segmentation methods in order to verify its ability to improve segmentation results. Comparisons were performed against traditional watershed, K-means clustering, and recursive shortest spanning tree (RSST) split and merge algorithm [22], using as quantitative, goodness criteria the Liu-Yang global cost (LYGC) [20] and Mumford-Shah energy functional (MSF) [21]. The smaller their values, the better the segmentation results are. Comparisons results are illustrated in Fig. 3, together with the corresponding quality measures. It is obvious that the proposed segmentation method outperforms the traditional watershed, Fig. 3(b). This was expected since the new scheme incorporates contrast and texture information, thus refining the watershed segmentation results by improving edge localization and suppressing boundaries at false edges (due to texture presence). If instead of $A(t)=1$ in the evolution PDE of (7) we use $A(t) \neq 1$ [10], which produces a volume flooding watershed-like term, the segmentation results are further improved in terms of better localization of region boundaries at the edges as can be seen in Fig. 3(a). The comparisons with RSST and K-means clustering (even after refinement and processing of the raw clustering results in order to create connected regions) shown Fig. 3(c) and (d) are in favor of the advanced scheme. The results of these methods appear undersegmented, and localization of the edges is poor and in the RSST case strongly quantized.

The coupled scheme, integrating textural and geometric information, yielded more meaningful segmentations compared to conventional methods and refined results with respect to classic watershed. Evaluation was performed both visually and by means of quality measures, for soil images of complex structure. The importance of texture analysis is revealed by the segmentation performance attained through the imagetexture decomposition scenario. Texture modulation energy also improves soil texture separation for classification, a fact that can be exploited for further analyzing segmented soil regions with precomputed features. A combination of geometric, statistical and textural measurements on the labeled images will map characteristic soil properties for the evaluation of its bioecological quality. Similar techniques could be applied to other aspects of subterranean and surficial analysis as well as to remote sensing imagery. To demonstrate this potential, we provide an aerial image example shown in Fig. 5, where a segmentation in homogeneous regions was achieved using the proposed methods.

\section{REFERENCES}

[1] R. Protz, M. Shipitalo, A. Mermut, and C. Fox, "Image-analysis of soilspresent and future," Geoderma, vol. 40, no. 1-2, pp. 115-125, Sep. 1987.

[2] P. M. C. Bruneau, D. A. Davidson, and I. C. Grieve, "An evaluation of image analysis for measuring changes in void space and excremental features on soil thin sections in an upland grassland soil," Geoderma, vol. 120, no. 3-4, pp. 165-175, Jun. 2004.

[3] P. Maragos et al., "Image analysis of soil micromorphology: Feature extraction, segmentation and quality inference," J. Appl. Signal Process., vol. 6, pp. 902-912, Jun. 2004.

[4] S. Beucher and F. Meyer, "The morphological approach to segmentation: The watershed transformation," in Mathematical Morphology in Image Processing, E. R. Doughertty, Ed. New York: Marcel Dekker, 1993.

[5] L. Vincent and P. Soille, "Watershed in digital spaces: An efficient algorithm based on immersion simulations," IEEE Trans. Pattern Anal. Mach. Intell., vol. 13, no. 6, pp. 583-598, Jun. 1991.

[6] L. Najman and M. Schmitt, "Watershed of a continuous function," Signal Process., vol. 38, no. 1, pp. 99-112, Jun. 1994.

[7] F. Meyer and P. Maragos, "Multiscale morphological segmentations based on watershed, flooding, and eikonal PDE," in Proc. Scale-Space'99 Conf., 1999, pp. 351-362.

[8] P. Maragos and M. A. Butt, "Curve evolution, differential morphology, and distance transforms applied to multiscale and eikonal problems," Fundamenta Inf., vol. 41, pp. 91-129, Jun. 2000.

[9] S. Osher and J. Sethian, "Fronts propagating with curvature-dependent speed: Algorithms based on Hamilton-Jacobi formulations," J. Comput. Phys., vol. 79, pp. 12-49, 1988.

[10] A. Sofou and P. Maragos, "PDE-based modeling of image segmentation using volumic flooding," in Proc. IEEE Int. Conf. Image Processing, vol. 2, 2003, pp. II-431-II-434.

[11] J. A. Sethian, Level Set Methods and Fast Marching Methods. Cambridge, U.K.: Cambridge Univ. Press, 1999.

[12] A. C. Bovik, N. Gopal, T. Emmoth, and A. Restrepo, "Localized measurement of emergent image frequencies by Gabor wavelets," IEEE Trans. Inf. Theory, vol. 38, no. 2, pp. 691-712, Mar. 1992.

[13] J. P. Havlicek, D. S. Harding, and A. C. Bovik, "Multidimensional quasieigenfunction approximations and multicomponent AM-FM models," IEEE Trans. Image Process., vol. 9, no. 2, pp. 227-242, Feb. 2000.

[14] P. Maragos and A. C. Bovik, "Image demodulation using multidimensional energy separation," J. Opt. Soc. Amer. A, vol. 12, no. 9, pp. 1867-1876, Sep. 1995.

[15] I. Kokkinos, G. Evangelopoulos, and P. Maragos, "Modulation-feature based textured image segmentation using curve evolution," in Proc. IEEE Int. Conf. Image Processing, vol. 2, 2004, pp. 1201-1204.

[16] L. A. Vese and S. J. Osher, "Modeling textures with total variation minimization and oscillating patterns in image processing," J. Sci. Comput., vol. 19, no. 1-3, pp. 553-572, Dec. 2003.

[17] F. Meyer and P. Maragos, "Nonlinear scale-space representation with morphological levelings," J. Vis. Commun. Image Represent., vol. 11, pp. 245-265, 2000.

[18] S. E. Grigorescu, N. Petkov, and P. Kruizinga, "Comparison of texture features based on Gabor filters," IEEE Trans. Image Process., vol. 11, no. 10 , pp. 1160-1167, Oct. 2002.

[19] M. Shi and G. Healey, "Hyperspectral texture recognition using a multiscale opponent representation," IEEE Trans. Geosci. Remote Sens., vol. 41, no. 5, pp. 1090-1095, May 2003.

[20] J. Liu and Y. Yang, "Multiresolution color image segmentation," IEEE Trans. Pattern Anal. Mach. Intell., vol. 16, no. 7, pp. 689-700, Jul. 1994.

[21] D. Mumford and J. Shah, "Optimal approximations by piecewise smooth functions and associated variational problems," Commun. Pure Appl. Math., vol. 42, no. 5, pp. 577-685, 1988.

[22] S. H. Kwok and A. G. Constantinides, "A fast recursive shortest spanning tree for image segmentation and edge detection," IEEE Trans. Image Process., vol. 6, no. 2, pp. 328-332, Feb. 1997. 